\title{
5G Associated Technologies and Challenges Worldwide
}

\author{
Maryam Nadeem and Siddhartha Sankar Biswas \\ \{maryam.nadeem20@yahoo.com and ssbiswas1984@gmail.com\} \\ Jamia Hamdard, New Delhi - 110062
}

\begin{abstract}
The fifth generation (5G) network is projected to support a huge amount of data traffic. It is meant to serve millions of wireless connections. With the utilization of certain advanced technologies like small cells, Massive MIMO, etc, 5G can support a wide range of devices and applications which will further give rise to an enhanced future of Internet of Things (IOT). However, these technologies have their own challenges that render the establishment of $5 \mathrm{G}$ difficult. In this paper, a brief overview of all these technologies along with their challenges are presented.
\end{abstract}

Keywords: 5G; Half Duplex FDD; Half Duplex TDD; Self-Interference Cancellation (SIC); OMA; Inter Modulation Distortion

\section{Introduction}

Not long ago, not even by 2016 could a large demography in India visualize the possession of a fast speed mobile network. It was only after the release of 4G LTE (that according to Wikipedia, was first publicly available in 2009) based smartphones that people have started having a hands-on experience of such a boon which within a blink of an eye has seen a surge. To everyone's surprise, it hasn't even entirely been a year where a greater section of people have been using the 4th generation mobile networks, that the 5 th generation is making way for itself in the market.

$5 \mathrm{G}$ is an opportunity to implement wired and wireless converged networks, and offers in particular opportunities in integrating network management systems [1,2]. You may refer to it as a beast in terms of mobile networks with a tremendous amount of enhancements in comparison to the previous generations, even 4G LTE, deploying improved technologies and ideas rendering a hoard of boosts mentioned as follows:

(i) FASTER: $5 \mathrm{G}$ provides up to 10 times faster speed than 4G LTE networks.

(ii) MORE TRAFFIC: It will be able to handle a 1000 times more traffic.

(iii) INCREASED BANDWIDTH: This aspect would allow millions of devices to be connected and hence lay foundation for virtual reality (VR), Internet of Things, autonomous driving, machine learning and more.

(iv) REDUCED LATENCY: It leads to a plunge in latency which goes down to $1 \mathrm{~ms}$. Evolution of mobile networks in terms of speed and latency has been shown in the following table 1: 
Table 1. Evolution of mobile networks in terms of speed and latency

\begin{tabular}{|l|l|l|l|l|l|}
\hline Year & 1980 & 1990 & 2000 & 2010 & 2020 \\
\hline Generation & $1 \mathrm{G}$ & $2 \mathrm{G}$ & $3 \mathrm{G}$ & $4 \mathrm{G}$ & $5 \mathrm{G}$ \\
\hline Speed & $2 \mathrm{kbps}$ & $384 \mathrm{kbps}$ & $56 \mathrm{MBps}$ & $1 \mathrm{GBps}$ & $10 \mathrm{GBps}$ \\
\hline Latency & N/A & $629 \mathrm{~ms}$ & $212 \mathrm{~ms}$ & $60.98 \mathrm{~ms}$ & $<1 \mathrm{~ms}$ \\
\hline
\end{tabular}

\section{Technologies And Challenges}

$5 \mathrm{G}$ has the ability to put forth these enhancements by making use of primarily five different technologies as mentioned in IEEE Spectrum. These technologies serve as the foundation for the emerging $5 \mathrm{G}$, emanating an immense growth in the services provided. However, there are various challenges adhered to some of them in combination with the $5 \mathrm{G}$ technology which restrict its establishment. These technologies along with the challenges are detailed below:

\subsection{Global Data Traffic Forecasted}

Traditionally, all the devices use only a certain range of frequencies under $6 \mathrm{GHz}$ on the radio frequency spectrum. And keeping in consideration today's need for more bandwidth due to the exponentially increasing number of devices and technologies like Wi-Fi, GPS, 4G, 3G and more that take up larger amount of data, it can be crystallized that this range is rapidly being filled up and is prone to causing delays. The global data traffic as was forecasted by Cisco is shown below in Figure 1:

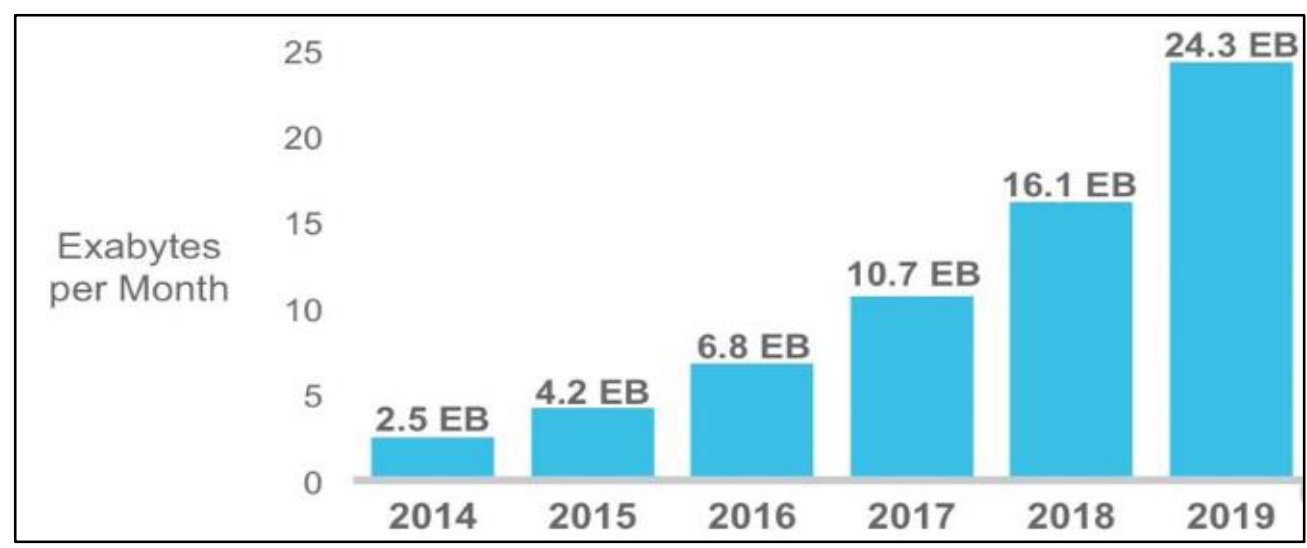

Fig. 1. Global data traffic as was forecasted by Cisco 


\subsection{Milimeter Waves}

Traditionally, a range that has a large amount of unused spectrum is necessitated to broadcast signals. In advocation of this, 5G uses millimeter waves called so because they have wavelengths lying between $1 \mathrm{~mm}$ and $10 \mathrm{~mm}$. These are high frequency waves ranging between $3 \mathrm{GHz}$ and $300 \mathrm{GHz}$ out of which the range from $24 \mathrm{GHz}$ to $100 \mathrm{GHz}$ (Figure 2) has been proposed for $5 \mathrm{G}$, and has the following advantages:

(i) This will allow more bandwidth to be allocated.

(ii) Higher frequency waves carry more data as compared to lower frequency waves.

(iii) Utilizing this part of the spectrum will make it possible to use massive MIMO antennas.

This will enable a huge amount of devices and technologies to be able to use the bandwidth as well as result in better streaming of higher quality videos and other multimedia content. However, this further exposes certain hindrances to $5 \mathrm{G}$.

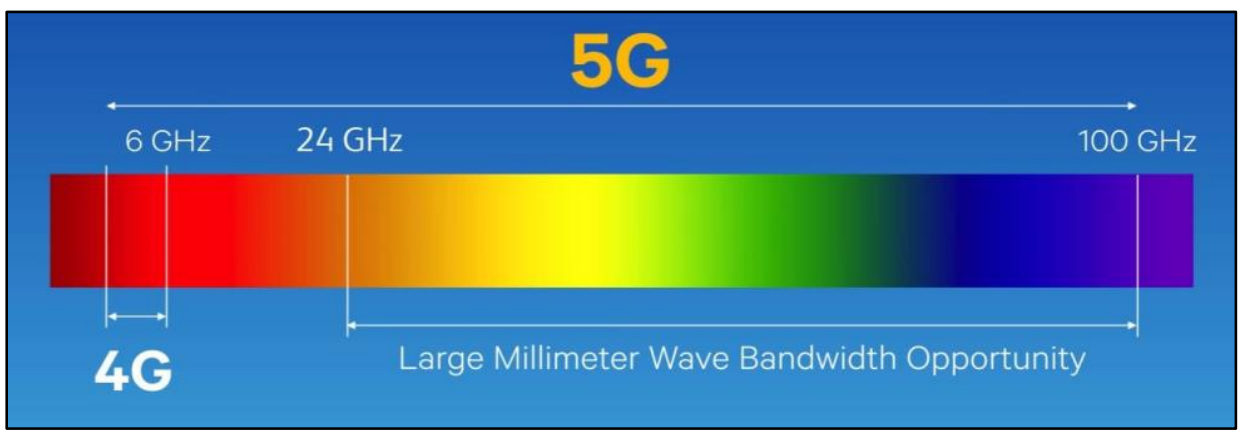

Fig. 2. Milimeter Waves range of $5 \mathrm{G}$

Since millimeter waves are high frequency waves, they are more susceptible to blockage from buildings, trees and other structures. Additionally, they can be attenuated by clouds and rains. For that reason, traditional cellular towers being used on a large scale today may render futile. It is so because the relationship between the wave frequency and antenna size is inversely proportional. The general rule is the higher the frequency, the smaller the antenna.

Frequency $\propto 1 /($ Antenna Size $)$

\subsection{Small Cell}

'Small cells' is a term for low powered radio access nodes that help in providing services to both indoor and outdoor areas. Small cell antennas have a range between $10 \mathrm{~m}$ to $2 \mathrm{~km}$. As explained earlier, macro antennas are potentially hopeless for millimeter waves, small cells cover up for this hurdle as they have defined purposes for providing end users with improvised cellular experience in congested urban areas. To extend the coverage of a macrocell, distributive antenna systems (DASs) are used in conjunction with the cell tower. DASs take a 
signal from the base station and boost it to increase the area the signal can reach. Small cells will be a crucial component for $5 \mathrm{G}$ networks, because they increase network capacity, density, speed and coverage.Using thousands of mini base stations that would be much closer together than the traditional towers will allow a better range of connection.Cells are divided into:
(i) Macrocell
(ii) Microcell
(iii) Picocell
(iv) Femtocell

Their coverage is shown below in Figure 3:

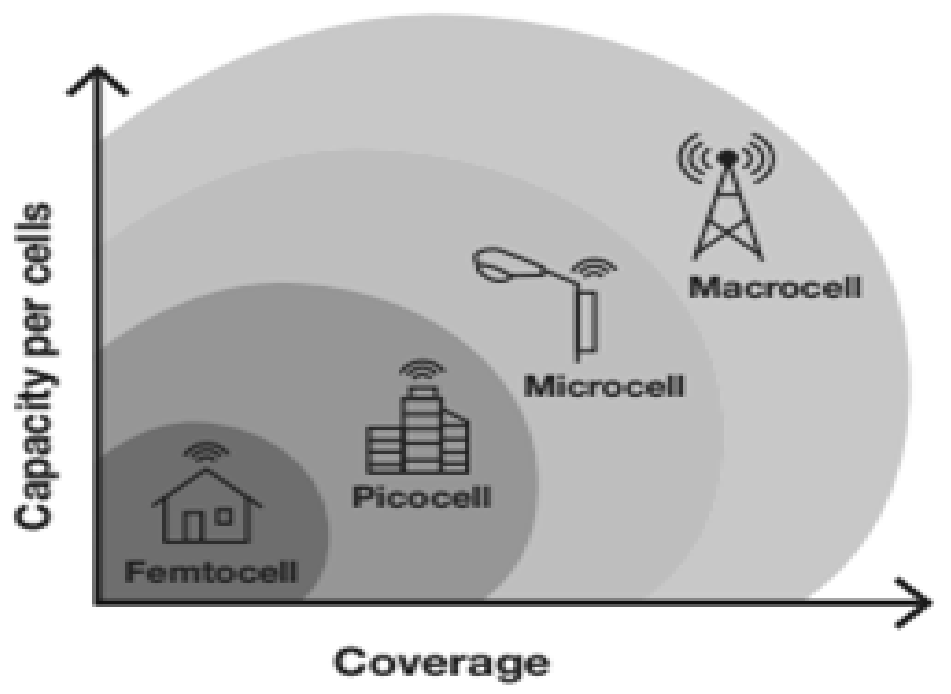

Fig. 3. Coverage by Small Cell

Apart from all the convenience provided by small cells, there are a handful of challenges or drawbacks while their implementation, them being:

(i) Small cells must be low cost because they are set up for fewer subscribers within a lesser range.

(ii) The number of small cells required to build a $5 \mathrm{G}$ network may make it hard to set up in rural areas.

(iii) It should be easy for the mobile network operator to diagnose potential problems and maintain small cells.

(iv) They should be physically small and lightweight in order to be deployed on streetlight poles, sides of building walls, etc.

(v) These should have high weather reliability.

(vi) Occurrence of interference is a greater possibility.

(vii) It will be a big challenge for $5 \mathrm{G}$ backhaul to support massive traffic and maintain the required quality of service with lower latency requirement. A solution has to be formulated on the physical layer. 
5G base stations will also have many more antennas than the base stations of today's cellular networks - to take advantage of another new technology: massive MIMO.

\section{Massive MIMO}

In wireless systems, the medium between the transmitter and receiver could be could be anything within the environment, and the transmitter has only a certain amount of power and bandwidth that it can use to transmit the data. Hence the signals are more prone to be attennuated and are disturbed by elements like trees, vehicles, etc which in turn is the cause of misinterpretation of the signal at the receiver's end. This is where massive MIMO comes into play.MIMO is a radio communication technolgy and stands for Multiple Input Multiple Output. As the basic framework of MIMO is to have multiple antennas at the transmitter and receiver (Refer to Figure 4). MIMO ensures reliable communication at high data rates as it takes advantage of the multiple paths that exist between the various transmitters and the receivers.

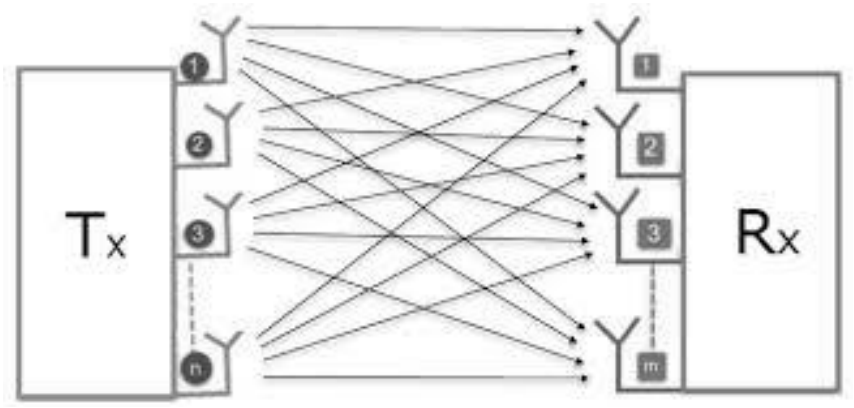

Fig. 4. Multiple Input Multiple Output

For older technologies, one cell can have only up to ten antennas but for 5G the very cell can have up to a hundred antennas this means one single cell can serve a lot more users at the same time and that with greater efficiency and speed. MIMO is already in operation on some 4G base stations but according to IEEE spectrum, massive MIMO has only been tested in labs and a few field trials and is seemingly a very promising technology for $5 \mathrm{G}$ as it increases throughput and has a higher capacity. But everything comes with a cost, this, meaning that massive MIMO possesses its own complications. Antennas broadcast information in all the directions at once, this could cause immense amount of interference. This issue can be solved by using another $5 \mathrm{G}$ technique called beamforming. 


\subsection{Beamforming}

Beamforming is a MIMO technique in which the transmitter or antenna focuses a narrow signal beam in the direction of the receiver. It requires that the transmitter knows the wireless channel. Multiple antennas placed in proximity, broadcasting a signal at slightly varied timings are deployed in the working of beamforming. The overlapping waves will produce constructive or destructive interference that will make the signal strong or weak respectively. If this is executed properly, beamforming focuses the signal to its path. The access point forms a narrow beam which has high gain in a specific direction, rather than across a wide angle. This beam points to the subscriber from which it has to receive data, intersecting with the subscriber's beam and receiving its data.This way beamforming eliminates the crisis of interference and imparts faster flow of data with fewer errors. It also saves the energy that the antenna generates as compared to conventional systems providing a higher signal level.

The limitations include the computing resources needed as they require more time and power. Beamforming computationally is the linear combination of the outputs of the elements, which a beam can be computed using the equation :

Where :

$$
\zeta(\theta)=\sum_{k=0}^{k=N} a_{k}(\theta) \cdot x_{k}
$$

- $\quad N$ is number of elements.

- $k$ is an index variable.

- $a_{k}$ is the complex coefficient of the $k^{\text {th }}$ element.

- $x_{k}$ is the voltage response from the $k^{\text {th }}$ element.

- $\zeta$ is the beam response.

- $\theta$ is the angle of the beam main lobe.

\subsection{Software Defigned Network (SDN)}

SDN can be introduced as a revolutionary approach rather than an evolutionary approach in the world of networking. (Refer to Figure 5) In legacy networks, devices like routers, switches, firewalls, etc have their own control planes called brains that communicate with neighboring devices, extract forwarding information and create databases [3,4]. Then algorithms are used to select the most optimum path from the databases and push these paths to the data plane called the body of the device. The actual forwarding of the packets to other ports is done by the data plane with the assistance provided by forwarding tables in the control plane. Each device has basically three layers:

(i) Hardware

(ii) Operating System

(iii) Application Layer

In a huge network, the managing of a large number of devices becomes a tedious task. In addition to this, when a network becomes more complex, policies such as firewalling policies, quality of service (QoS), etc tend to get piled up. Hence, updating these policies manually becomes nearly impossible as in a distributed network it takes a long amount of time. Moreover, maintaining consistency between routers or other devices becomes difficult. 
The burden would be much less if this was accomplished automatically, and this is just where SDN plays a vital role. In SDN, the control plane is segregated from its respective data plane physically i.e. the application and OS layer are separated from the hardware centralizing its intelligence and abstracting its architecture. A single control plane is made up of all the

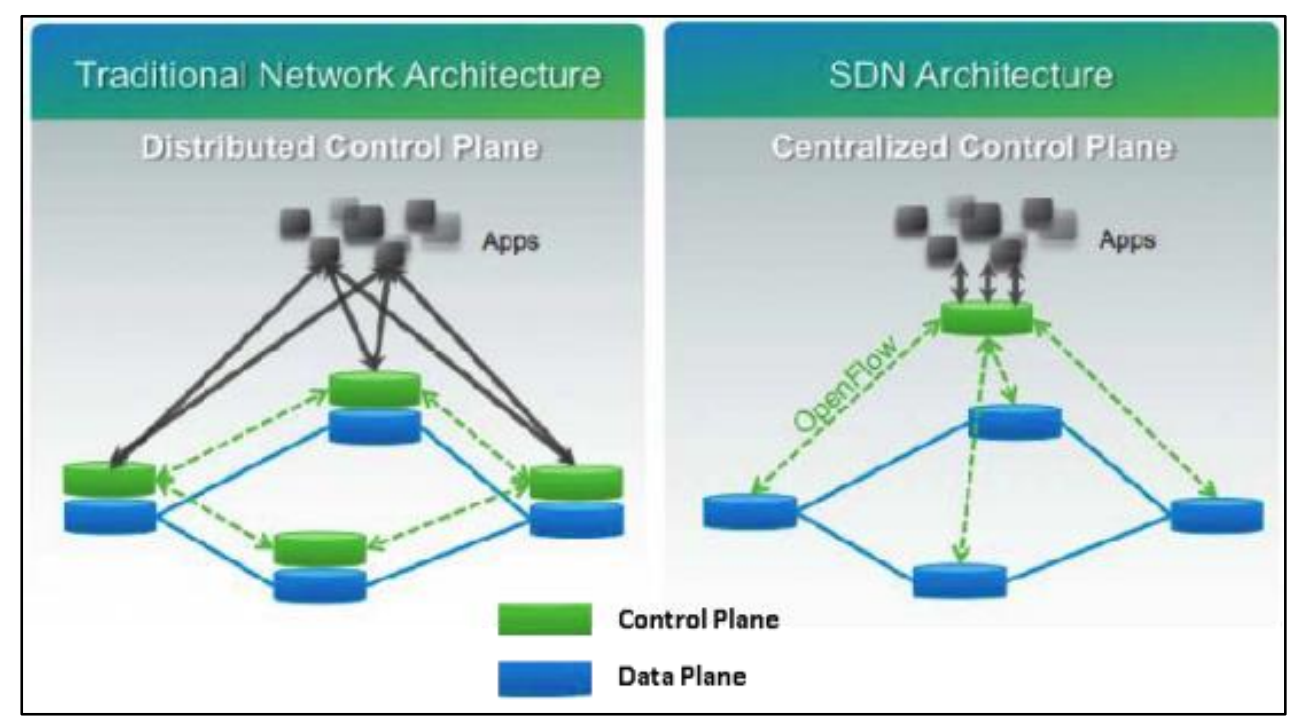

individual control planes and it does exactly the same job as earlier, just for a larger number of devices as a whole which means that there is a defined control logic in a centralized manner. Communication between the two planes is done through APIs. For making controller to switch communication, protocols like OpenFlow can be used.

Fig. 5. Software Defigned Network (SDN)

SDN is fruitful also because the devices become much cheaper now with the elimination of the control planes. There are several SDN models like Cisco ACI. The goal of all SDN models is to make the networks more agile and manageable. There are two main challenges in the implementation of SDN:

1. Rule Placement Problem:

(i) Forwarding in SDN is done using flow tables defined by the centralized controller.

(ii) The size of the memory called ternary content addressable memory (TCAM) is limited.

(iii) In addition to this TCAM is very expensive.

(iv) At times there's a 3-5 ms delay in a new rule placement.

2. Controller Placement Problem:

(i) Controllers define flow rule according to the requirements and must be able to handle all the requests which becomes prone to causing delays.

(ii) If we have very less controllers for a large network, it might get congested. 


\section{Conclusion}

Regardless of the most advanced technologies ready to be brought into service by the fifth generation network, there are significant numbers of other challenges that thwart the successful establishment of $5 \mathrm{G}$ worldwide. These are listed below:

(i) The lack of coordination of frequencies worldwide. Different countries are observed to have different frequencies.

(ii) Making calibrated measurements of frequencies and bandwidth is very expensive, time-consuming and requires a lot of expertise.

(iii) Inter Modulation Distortion (IMD) could be caused due to the proximity of the bands of 5 G NR and legacy LTE systems.

(iv) The ability to cover wide ranges of geographic spectrum and fitting into the footprints of today's cellular network with high frequency ranges is a big challenge within itself.

(v) Management of power consumption i.e. generation and dissipation of power in antennas and other such devices.

(vi) Reports estimate the cost to deploy a small cell-ready 5G network - assuming fibre backhaul is commercially feasible - can range from USD 6.8 million for a small city to USD 55.5 million for a large, dense city

(vii) Rural and suburban areas are less likely to enjoy $5 \mathrm{G}$ investment, and this will potentially widen the digital divide.

Expectations of $5 \mathrm{G}$ are high, with many assuming it will deliver a transformative promised land - an improved end-user experience, new applications, new business models and new services riding swiftly on the back of gigabit speeds, improved network performance and reliability. 5G networks and services, standing as they do on the shoulders of successful $2 \mathrm{G}$, $3 \mathrm{G}$ and $4 \mathrm{G}$ mobile networks, are forecast by independent economic studies to deliver very significant economic gains by this decade.

\section{References}

[1] Aleksandar Tudzarov and Toni Janevski, "Functional Architecture for 5G Mobile Networks" InternationalJournal of Advanced Science and Technology Vol. 32, July, 2011.

[2] Ms. Reshma S. Sapakal, Ms. Sonali S. Kadam, "5G Mobile Technology” International Journal ofAdvanced Research in Computer Engineering \& Technology (IJARCET) Volume 2, Issue 2, February2013.

[3] Poornima, M., Kumar, V., \& Singh, R. K. (2018). A Review Paper on 5G Wireless Technology. Journal of Instrumentation and Innovation Sciences, 3(2).

[4] Gohil, A., Modi, H., \& Patel, S. K. (2013, March). 5G technology of mobile communication: A survey. In Intelligent Systems and Signal Processing (ISSP), 2013 International Conference on (pp. 288-292). IEEE. 